\title{
Copro-antigen capture ELISA for the detection of Teladorsagia (Ostertagia) circumcincta in sheep: improvement of specificity by heat treatment
}

\author{
D. A. JOHNSON${ }^{1}$, J. M. BEHNKE ${ }^{1 *}$ and G. C. COLES ${ }^{2}$ \\ ${ }^{1}$ School of Biology, University of Nottingham, University Park, Nottingham NG7 2RD, UK \\ ${ }^{2}$ Department of Clinical Veterinary Science, University of Bristol, Langford House, Bristol BS40 5DU, UK
}

(Received 25 September 2003; revised 23 December 2003; accepted 7 Fanuary 2004)

SUMMARY

A copro-antigen capture ELISA for the detection of Teladorsagia circumcincta infection in sheep was developed and evaluated. Experiments with faeces from worm-free sheep, that had been spiked with known concentrations of excretory-secretory (E-S) antigen indicated that a positive signal was generated with $180 \mathrm{ng}$ of E-S/ml. A nested design, based on 8 infected and 8 worm-free sheep, was employed to assess the stages during sample preparation contributing to variation in signal from the assay. This showed that $87 \%$ of the variance in the optical density readings (ODs) was directly explained by infection status. Variation between individual sheep within infection groups, and between samples at various stages in the assay, collectively accounted for the remaining variance. Initial evaluation of specificity using faeces from animals carrying a range of monospecific infections indicated cross-reactivity with Haemonchus contortus and Nematodirus spathiger. However, by treating the supernatant from faeces for $20 \mathrm{~min}$ at $100{ }^{\circ} \mathrm{C}$, the cross-reactive signal was eliminated whilst the specific signal was largely preserved. Heat treatment of faeces from 12 non-infected sheep, 12 sheep with $T$. circumcincta and 6 with $H$. contortus resulted in sensitivity being increased from $66 \cdot 7$ to $85 \cdot 7 \%$, and specificity from $62 \cdot 5$ to $87 \cdot 5 \%$. OD values showed a significant positive relationship with adult worm burdens, although at low infection intensities there was some overlap between infected and worm-free animals. We discuss the application of CC-ELISAs in facilitating selective chemotherapy of sheep, as a means of avoiding the development of anthelmintic resistance in pastoral regions where sheep are farmed on a large scale.

Key words: copro-antigen ELISA, Teladorsagia (Ostertagia) circumcincta, sheep, nematode, excretory-secretory (E-S) products, antibodies.

\section{INTRODUCTION}

With the increasing spread of anthelmintic resistance among the gastro-intestinal (GI) nematodes of domestic animals (Waller, 1993; Waller et al. 1995 ; Hennessy, 1997), particularly multiple resistance (Jackson, Jackson \& Coop, 1992), and the increasing consumer demand for reduced levels of drug residues in food and the environment (Stear \& Murray, 1994), new approaches to control are urgently required. The traditional, and most widely implemented, control measure for GI nematodes is mass chemotherapy at regular times throughout the year, a treatment regimen that encourages the development of anthelmintic resistance (Waller, 1986; Coles, 1995 ; Condor \& Campbell, 1995; Jackson \& Coop, 2000). One approach that might reduce the onset and spread of resistance would be to replace mass chemotherapy with selective chemotherapy, focussing treatment only on those animals that require drenching because

* Corresponding author: School of Biology, University of Nottingham, University Park, Nottingham NG7 2RD. Tel: +44(0) 1159513208 . Fax: + 44 (0) 1159513251. E-mail: jerzy.behnke@nottingham.ac.uk of worm burdens that threaten production traits if left untreated (Smith, 1990). However, accurate detection of infection, particularly in animals carrying subclinical infections, is essential if selective chemotherapy is to be rationally implemented. For the most part, diagnosis of infection with GI nematodes is still dependent on relatively expensive monitoring of faecal egg counts (FEC) by microscopy, with the ensuing disadvantages that the methods are laborious, time consuming, unreliable, incapable of detecting pre-patent infections and dependent on skilled microscopists (Nicholls \& Obendorf, 1994; Johnson, 1999; Rodriguez-Canul et al. 1999). Although a kit for use by farmers is available and has been promoted in New Zealand and elsewhere, FEC are still not practical for individual livestock owners and in practice are largely based in reference laboratories run by national or private agricultural advisory organizations (e.g. DEFRA, formerly MAFF in the UK).

Automated detection of GI worm infections, and particularly the distinction between animals not infected or carrying low intensity parasite burdens and those with heavy infections, would allow focused treatment with anthelmintics. It is the latter group, 
animals carrying the heaviest infections, that poses most concern to farmers and merits priority in treatment. However, this approach can only be economically viable in regions of the world where sheep farming is profitable and only if cheap and rapid assays for GI nematode infections were to be available. In principle, copro-antigen capture enzyme-linked immunoabsorbent assays (CC-ELISA; Green, 1986) can provide the necessary means to facilitate targeted anthelmintic treatment. These assays are useful diagnostic tools because they reflect current intestinal infection with adult worms (Allan et al. 1992; Deplazes et al. 1992) and moreover they are fast, sensitive and relatively easy to use, eliminating the need for veterinary assistance and trained microscopists (Johnson et al. 1994). CC-ELISAs have been very successful commercially in detecting protozoan infections (Green, Warhurst \& Miles, 1985 ; Baumann \& Gottstein, 1987; Jelenik et al. 1996) and have been shown to be feasible for the detection of helminth, notably cestode, infections (Allan et al. 1996, 1992). Moreover, they have been adapted recently for the detection of GI nematodes in both laboratory model systems (Nageswaren, Craig \& Devaney, 1994; Johnson, Behnke \& Coles, 1995) and in domestic animals (Ellis et al. 1993; Roepstorff, 1998; Agneessens, Claerebout \& Vercruysse, 2001).

In our earlier paper we demonstrated the feasibility of a CC-ELISA for GI nematodes with the model system Heligmosomoides polygyrus in mice (Johnson et al. 1995). Johnson (1994) and Johnson et al. (1999) presented preliminary results indicating that an assay for Teladorsagia circumcincta, quantitatively the most important GI nematode of sheep in the UK and in northern pastoral regions of the world (Urquhart et al. 1987), was also feasible. Since then an assay for the related species Ostertagia ostertagi in cattle has been published (Agneessens et al. 2001). In this paper we evaluate a CC-ELISA for the detection of $T$. circumcincta, our main objective being to confirm proof of principle and to establish that such an assay for T. circumcincta is feasible and scientifically valid. When our initial efforts were frustrated by cross-reactivity with other common ovine GI nematodes, we developed a novel heat-based treatment that almost completely eliminated the crossreactivity whilst largely preserving the strength of the specific signal and in particular the signal to noise ratio.

\section{MATERIALS AND METHODS}

\section{Isolation and recovery of adult}

Teladorsagia circumcincta

The abomasum was isolated from freshly slaughtered sheep, cut longitudinally, and washed in Hanks' Balanced Salt Solution (HBSS). Worms were recovered from the abomasum by a modification of the Baermann technique based on placing the abomasum on a $300 \mu \mathrm{m}$ sieve and incubation in HBSS at $37{ }^{\circ} \mathrm{C}$ to facilitate isolation of worms. Worms were washed 5 times in sterile $0 \cdot 1 \mathrm{M}$ phosphate-buffered saline $(\mathrm{PBS}), \mathrm{pH} 7 \cdot 4$, containing $1 \%(\mathrm{w} / \mathrm{v})$ glucose, $500 \mathrm{IU} / \mathrm{ml}$ penicillin and $5 \mathrm{mg} / \mathrm{ml}$ streptomycin, in preparation for in vitro culture.

In experiments where the total worm burden was required, the incubation period was increased to $6 \mathrm{~h}$ to ensure full recovery of worms - these worms were not used for the production of Excretory-Secretory (E-S) products. After $6 \mathrm{~h}$ the abomasum was removed from the sieve, which was then carefully scrutinized for any remaining worms. Worms were recovered from the incubation solution and counted immediately. The abomasal washings were made up to 1.5 litres with $\mathrm{HBSS}$, mixed well, and a $100 \mathrm{ml}$ aliquot removed, to which $5 \mathrm{ml}$ of Lugols' iodine were added to kill/stain the worms. Aliquots were examined microscopically for worms, and the final worm burden was calculated.

\section{Preparation of T. circumcincta $E-S$ products}

Freshly isolated worms were washed further with sterile RPMI 1640 (Gibco) containing glucose (1\% $\mathrm{w} / \mathrm{v})$, gentamycin $(1 \mu \mathrm{g} / \mathrm{ml})$, penicillin/streptomycin $(500 \mathrm{IU} / \mathrm{ml}$ and $5 \mathrm{mg} / \mathrm{ml})$ and glutamine $(10 \mu \mathrm{M})$, transferred to a sterile culture flask and incubated at a density of approximately 100 worms $/ \mathrm{ml}$ of medium. Cultures were maintained at $37{ }^{\circ} \mathrm{C}$ for $14 \mathrm{~h}$, after which the supernatant was removed and filtered through a $0 \cdot 22 \mu \mathrm{m}$ Millipore filter, to eliminate parasite eggs. The supernatant was then dialysed against distilled water over a period of $24 \mathrm{~h}$, and concentrated approximately 50-fold, using Centricon-10 filters (Amicon, UK). Protein content was estimated by the Bradford method (Bradford, 1976) and the material stored at $-80{ }^{\circ} \mathrm{C}$.

\section{Rabbit polyclonal antisera}

Adult female New Zealand White rabbits were supplied by the Queens Medical Centre Animal House, University of Nottingham and were maintained under conventional conditions in the School of Biology Animal House. Following collection of naïve blood samples from each rabbit (approximately $5 \mathrm{ml}$ ), rabbits were vaccinated according to the following protocol, each boost being administered at 2-weekly intervals. The primary dose, consisting of $300 \mu \mathrm{g} T$. circumcincta $\mathrm{E}-\mathrm{S}$ antigen, in a volume of $1 \mathrm{ml}$ of PBS, was emulsified with $1 \mathrm{ml}$ of Freund's Complete adjuvant (SIGMA) and injected subcutaneously in 4 sites. The first boost comprised $150 \mu \mathrm{g} \mathrm{T}$. circumcincta $\mathrm{E}-\mathrm{S}$ antigen, in a volume of $1 \mathrm{ml}$ of PBS, emulsified with $1 \mathrm{ml}$ of Freund's Incomplete adjuvant (SIGMA) and this was also injected subcutaneously in 4 sites. An identical protocol was 
followed for the second and third boosts. One day prior to each boost a 1-2 ml blood sample was taken from the lateral ear vein in order to test the serum antibody levels during the vaccination period.

Two weeks following the final boost, the rabbits were exsanguinated. Blood was left to clot for 1-2 h at room temperature, ringed to separate the clot from the wall of the universal tube, and left to clot further overnight at $4{ }^{\circ} \mathrm{C}$. The fluid was subsequently removed, and centrifuged at $2000 \mathrm{~g}$ for $10 \mathrm{~min}$, after which the serum was collected and stored in aliquots at $-80{ }^{\circ} \mathrm{C}$.

\section{Isolation and labelling of rabbit IgG from whole rabbit serum}

A $5 \mathrm{ml}$ volume of whole serum was used for each $\mathrm{IgG}$ isolation. The $\mathrm{pH}$ of the serum was adjusted to pH 7.0, by dialysis against $0.02 \mathrm{M}$ sodium phosphate buffer (pH 7-0). A Hi Trap G (Protein G) column (Pharmacia) was washed out twice with $15 \mathrm{ml}$ of distilled water, in order to remove the ethanol preservative. The column was then equilibrated with 3 column volumes of $0.02 \mathrm{M}$ sodium phosphate buffer (pH 7.0). The serum sample was added to the column, with a syringe, and the column was washed with 5 column volumes of $0.02 \mathrm{M}$ sodium phosphate buffer $(\mathrm{pH} \mathrm{7 \cdot 0)}$. IgG was then eluted with $0 \cdot 1 \mathrm{M}$ glycine- $\mathrm{HCl}$ buffer ( $\mathrm{pH} 2 \cdot 7)$. Fractions of $1 \mathrm{ml}$ were collected into Eppendorf tubes containing $100 \mu \mathrm{l}$ of $1 \mathrm{~m}$ Tris-HCl buffer ( $\mathrm{pH} 9 \cdot 0)$, to ensure that the final $\mathrm{pH}$ of the sample was neutral. The fractions were analysed on a spectrophotometer at $280 \mathrm{~nm}$, and the fractions containing $\mathrm{IgG}$ were pooled, and subsequently concentrated to $7-8 \mathrm{mg} / \mathrm{ml}$, using Centricon 10 filters (Amicon, UK). Aliquots were stored at $-80{ }^{\circ} \mathrm{C}$. The Hi Trap columns were stored in $20 \%$ ethanol at $4{ }^{\circ} \mathrm{C}$.

Conjugation of alkaline phosphatase to rabbit IgG was carried out using a kit manufactured by Boehringer Mannheim (Catalogue no. 1829688 ). The $\mathrm{pH}$ of the $\mathrm{IgG}$ solution was adjusted to $\mathrm{pH} 9 \cdot 8$, by overnight dialysis against $1 \mathrm{M}$ sodium carbonate buffer ( $\mathrm{pH} 9 \cdot 8$ ). The concentration of the IgG solution was checked photometrically prior to coupling, to ensure a critical concentration of $6-8 \mathrm{mg} / \mathrm{ml}$. Fifty $\mu$ l of the IgG solution were added to $100 \mu \mathrm{l}$ of alkaline phosphatase $(20 \mathrm{mg} / \mathrm{ml})$, mixed well, and incubated overnight at $4{ }^{\circ} \mathrm{C}$. Twenty $\mu$ l of triethanolamine solution $(2 \mathrm{M}, \mathrm{pH} 8 \cdot 0)$ were added to the mixture, followed by $40 \mu \mathrm{l}$ of sodium borohydride solution $(200 \mathrm{~mm})$, mixed well, and incubated at $4{ }^{\circ} \mathrm{C}$ for $30 \mathrm{~min}$. Five $\mu \mathrm{l}$ of triethanolamine solution $(2 \mathrm{M}$, $\mathrm{pH} 8.0$ ) were added, and the mixture incubated again for $2 \mathrm{~h}$ at $4{ }^{\circ} \mathrm{C}$. The conjugate was stabilized by the addition of $10 \mu \mathrm{l}$ of glycine solution $(1 \mathrm{M}, \mathrm{pH} \mathrm{7 \cdot 0)}$ to the incubation solution, and mixed well. The solution was dialysed overnight with 3 changes of $200 \mathrm{ml}$ of dialysis buffer (pH 6.5; Manufacturer's own). The conjugate was then transferred to an Eppendorf tube, the volume determined, and the same volume of stabilizing reagent, with BSA and Kathon ${ }^{\circledR} \mathrm{CG}$ (Manufacturer's own) was added. The conjugate was stored at $4{ }^{\circ} \mathrm{C}$.

\section{Coproantigen ELISA for T. circumcincta}

All the procedures described here were optimized and have been described fully by Johnson (1999). These include optimal concentrations of capture and detection antibodies, faecal supernatant dilution, blocking agents, times for incubation and microtitre plates. The description that follows is based on optimal concentrations and times, with just one exception. The role of heat treatment in reducing crossreactivity is evaluated and the optimal treatment described and justified in the results section of this paper. Other approaches for reducing crossreactivity were also evaluated, but none of these proved as useful as heat treatment (Johnson, 1999).

A $5 \mu \mathrm{g} / \mathrm{ml}$ solution of rabbit anti- $T$. circumcincta ES IgG was prepared in $1 \mathrm{M}$ carbonate-bicarbonate buffer ( $\mathrm{pH} 9 \cdot 6$ ), and $50 \mu \mathrm{l}$ added to each well of 96-well flat-bottomed Dynatech Immulon 1 plates (capture antibody). The plates were sealed with cling-film, incubated at $4{ }^{\circ} \mathrm{C}$ overnight and then washed 3 times with PBS $0.05 \%$ Tween 20 . In order to block unoccupied binding sites, $100 \mu \mathrm{l}$ of $1 \%$ (w/v) bovine serum albumin (BSA; Fraction V, Sigma) in PBS $0.05 \%$ Tween 20 were added to each well, and left for $1 \mathrm{~h}$ at room temperature. Plates were washed 3 times with PBS 0.05\% Tween 20 .

Faecal supernatant was prepared by mixing the faecal sample with PBS $0 \cdot 3 \%$ Tween 20 , at a dilution of $1: 200$, until faecal slurry was formed. The mixture was then centrifuged at $2000 \boldsymbol{g}$ for $30 \mathrm{~min}$. Fifty $\mu \mathrm{l}$ of the supernatant were added to each well of the microassay plate, and incubated for $90 \mathrm{~min}$ at room temperature. All samples were run in triplicate. Plates were washed 3 times with PBS 0.05\% Tween 20 .

Fifty $\mu 1 /$ well of a $1: 160$ dilution of rabbit anti- $T$. circumcincta ES IgG alkaline phosphatase were added (detecting antibody), and the plates incubated for $90 \mathrm{~min}$ at room temperature. Plates were washed 5 times with PBS $0.05 \%$ Tween 20, the last 2 washes consisting of $1 \mathrm{~min}$ incubations. Then $100 \mu \mathrm{l}$ of substrate solution ( $\mathrm{p}$-nitrophenol phosphate disodium, Sigma Fast) were added to each well, and plates incubated in the dark for $30 \mathrm{~min}$ at $37^{\circ} \mathrm{C}$. Optical density readings were taken at $405 \mathrm{~nm}$ on a Dynatech microassay reader.

\section{Procedures used in evaluating the assay}

The sheep from which adult parasites, and faecal samples were collected, were generously made available by the Moredun Research Institute ( $T$. circumcincta, T. colubriformis, N. battus and material 
from worm-free animals), Bristol Veterinary School (T. circumcincta, H. contortus), Veterinary Laboratory Agency (Department of the Environment, Food and Rural Affairs, HM Government, UK; N. spathiger), Pfizer Animal Health ( $C$. oncophora) and Compton Animal Health (material from worm-free sheep). We were dependent on the suppliers for the breed, age and sex of the sheep, and the isolates of ovine parasites used since the samples provided were mostly from animals involved in various trials at the respective research centres.

\section{Evaluation of the sensitivity of the assay}

In order to evaluate the capture sensitivity of the assay in detecting $T$. circumcincta $\mathrm{E}-\mathrm{S}$ antigens in ovine faecal material, uniform volumes of E-S products were added to faecal supernatants, prepared from faecal samples from worm-free sheep (Moredun barn-reared lambs). To control for interfering components of the faeces, E-S products were also prepared with $\mathrm{PBS}$, to the same uniform volumes, of known concentrations. The E-S products were added to faecal supernatant or PBS in doubling dilutions in order to give a final range of concentrations of E-S from $0 \cdot 0625 \mu \mathrm{g} / \mathrm{ml}$ to $8 \mu \mathrm{g} / \mathrm{ml}$.

\section{Evaluation of the range of signal from non-infected animals}

In order to determine the range of background, or noise level, in the ELISA, and thereby to provide a cut-off value above which the ELISA signal could be considered to come from infected animals, faecal samples from 98 worm-free, barn-reared lambs were assayed. These were compared with 8 samples from animals experimentally infected with $T$. circumcincta.

\section{Assessment of inter- and intra-sample variation in optical density readings}

The degree of variation between, and within faecal samples taken from sheep infected with $T$. circumcincta and samples taken from worm-free sheep, was tested under standard optimum conditions. Faecal samples from 8 sheep experimentally infected with $T$. circumcincta and 8 worm-free sheep were used for this purpose. Each faecal sample was divided into 3 portions, from each of which 3 faecal supernatants were prepared independently. Each faecal supernatant preparation was then tested in triplicate.

\section{Assessment of the cross-reactivity of the assay}

Faecal supernatants were prepared from sheep harbouring moderate patent infections of Haemonchus contortus $(n=3), \quad$ Trichostrongylus colubriformis $(n=2)$, Cooperia oncophora, Nematodirus battus $(n=2)$ and Nematodirus spathiger. Also tested in the assay were faecal samples from mice experimentally infected with Heligmosomoides polygyrus $(n=4)$, Trichinella spiralis $(n=2)$ and Hymenolepis microstoma $(n=3)$, and faecal samples from hamsters infected with Necator americanus $(n=4)$. All infections were confirmed through the presence of eggs in the faeces. These were compared to faecal samples from 4 sheep infected with $T$. circumcincta and from 5 worm-free sheep. Faecal samples were evaluated for individual animals, and mean values based on triplicate ODs for each animal were used to calculate a group mean ( \pm S.E.M.).

\section{Evaluation of sensitivity and specificity of the assay}

To estimate conventional diagnostic sensitivity and specificity of the assay we used the formulae given by Galen \& Gambino (1975) where

sensitivity $=$

$\frac{\text { true positive results }}{\text { true positive results }+ \text { false negative results }} \times 100$,

and

specificity $=$

true negative results $\times 100$.

true negative results + false positive results

Relationship between $O D$ signals generated in the coproantigen ELISA and the worm burden

In order to relate the signal produced in ELISA to the worm burdens of infected animals, abomasa and faecal samples were collected from freshly slaughtered lambs at a local abattoir. Several visits were made to the abattoir in 1999 until a total of 23 sheep had been sampled. Faecal supernatants were prepared from the faecal sample of each animal, heat treated, and then tested in triplicate, under optimal conditions in the ELISA. Worms were recovered from the abomasa as described earlier and the numbers of T. circumcincta present were counted.

\section{Data presentation and statistical analysis}

Some of the data are derived from single samples, and for these the values presented are means of triplicate assays. Where quantitative data were employed, these are summarized as mean values \pm standard error of the mean (S.E.M.). Quantitative data were analysed by ANOVA, by repeated measures ANOVA or by nested ANOVA, depending on the format of the data and the required analysis, using the software packages SPPS version 9.0.0 or Statgraphics Version 7. Correlations between variables 


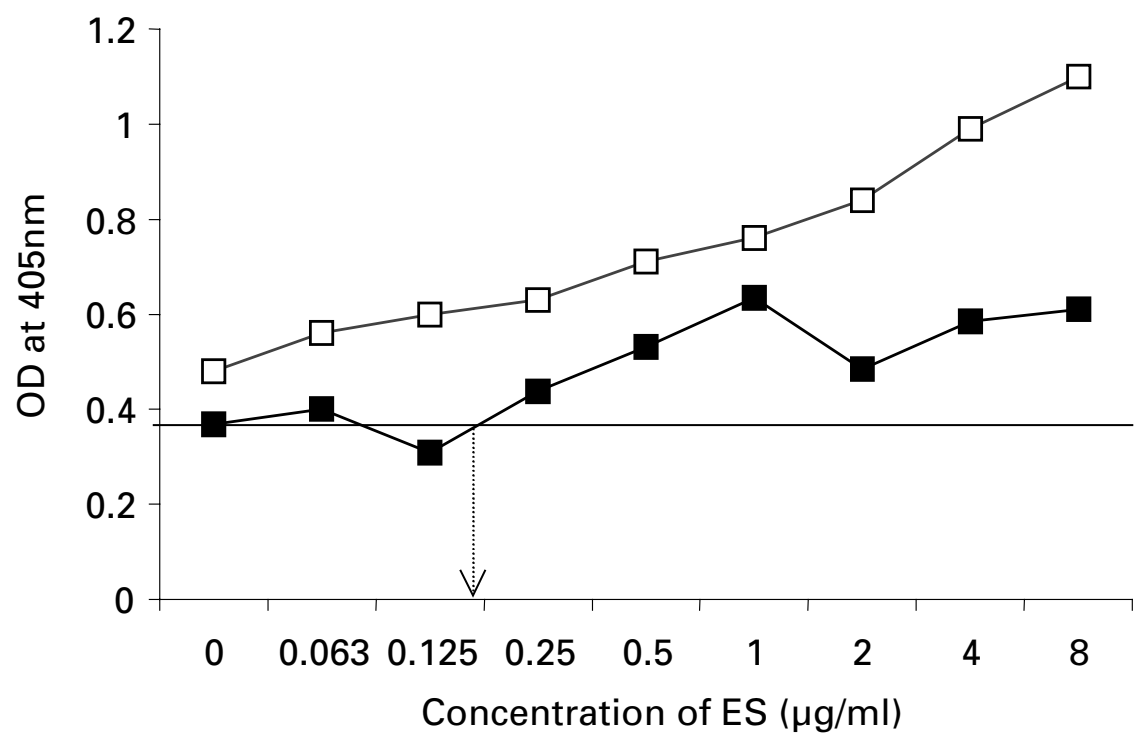

Fig. 1. Sensitivity of the copro-antigen capture ELISA, with PBS $(\square)$ or faecal supernatant from non-infected sheep (ם) as diluents. E-S products were added in equal volume to PBS or supernatant from faeces from non-infected sheep in doubling dilutions, as described in the text. The mean OD (+3 s.D.) of the unspiked faecal supernatant is indicated by the horizontal line. The vertical arrow indicates the lowest concentration of antigen detected by the assay.

were assessed by Spearman's Test based on ranks. We employed a stringent threshold for the detection of infection, equivalent to an upper limit for values from worm-free control animals, calculated in each run of the assay as the mean value plus 3 times the standard deviation (S.D.). This should cover about $99 \%$ of the values from uninfected animals and values above this were considered to indicate the presence of infection.

\section{RESULTS}

\section{Capture sensitivity of the assay}

The sensitivity of the assay in detecting low levels of $T$. circumcincta $\mathrm{E}-\mathrm{S}$ antigens added to the supernatant extract of ovine faeces is illustrated in Fig. 1. As can be seen, the signal produced in the ELISA increased consistently as the concentration of E-S increased in both faecal supernatant (FSN) and PBS, although a stronger signal was detected in the case of E-S diluted with PBS, compared with FSN. The results obtained show a lower resolving limit of approximately $0 \cdot 18 \mu \mathrm{g} / \mathrm{ml}(180 \mathrm{ng} / \mathrm{ml})$ for E-S antigen in sheep faeces. There was a significant positive correlation between $\mathrm{OD}$ and the concentration of E-S in both cases (PBS $r_{s}=0 \cdot 917, n=9, P<0.001$; FSN $\left.r_{s}=0 \cdot 634, n=9, P=0 \cdot 005\right)$.

\section{Determination of a diagnostic cut-off value}

Fig. 2 shows that the values obtained with samples from worm-free sheep were closely grouped and typically with low ODs (range 0.01-0.02). The respective cut-off value was calculated as 0.078 . All the samples from infected animals were above this level, although three samples from worm-free sheep also exceeded the threshold for non-infected animals, giving a $100 \%$ accuracy in diagnosis of infected sheep and $96.9 \%$ accuracy for worm-free status. The sample from infected sheep Red 6, registered a low OD of $0 \cdot 12$, just above the threshold. This particular sample was 13 months old, and could have deteriorated (see below).

\section{Assessment of inter-and intra-sample variation in optical density readings}

In order to assess the extent of variation within, and between samples, and at the various stages during preparation of samples for assay, the OD values obtained from 8 infected and 8 worm-free sheep were analysed by Nested ANOVA and the results are summarized in Table 1 . The majority of variance in the data was attributable to the difference between infected and worm-free status. Inter-sample variation, among $T$. circumcincta and worm-free groups, accounted for just $12 \%$ of the variance and withinsample variance was negligible. A further $0 \cdot 32 \%$ of variance was attributable to variation between supernatant preparations and $0 \cdot 37 \%$ remained unexplained.

\section{Assessment of the cross-reactivity of the assay}

The results in Fig. 3 show that faecal material from sheep infected with $T$. circumcincta generated a high OD reading compared to the cut-off value of $0 \cdot 19$ derived from worm-free sheep. The signal: noise ratio was 5 . However, it is also evident that there was a significant amount of cross-reactivity with $H$. contortus (signal : noise ratio $4 \cdot 2$ ) and $N$. spathiger 
Table 1. Assessment of inter- and intra-sample variation in OD signal by nested ANOVA of optical density readings from faecal samples obtained from worm-free and infected sheep

\begin{tabular}{lrrrr}
\hline \hline $\begin{array}{l}\text { Source of } \\
\text { variation }\end{array}$ & D.F. & $\begin{array}{l}\text { Mean } \\
\text { square }\end{array}$ & $\begin{array}{l}\text { Variance } \\
\text { component }\end{array}$ & Percentage \\
\hline Infection status* & 1 & 228.99 & $1 \cdot 042$ & 87.07 \\
Sheep广 & 14 & 3.96 & 0.146 & 12.24 \\
Faecal samplet & 32 & 0.015 & $<0.001$ & $<0.01$ \\
Faecal supernatant $\$$ & 96 & 0.016 & 0.004 & 0.32 \\
Error & 288 & 0.004 & 0.004 & 0.37 \\
\hline \hline
\end{tabular}

* Samples were either from infected or worm-free sheep.

$\dagger$ Samples were assayed from 8 infected and 8 worm-free sheep.

* Each faecal sample was divided into 3 subsamples that were processed independently.

$\S$ Each faecal subsample provided 3 separate supernatants for the assay.

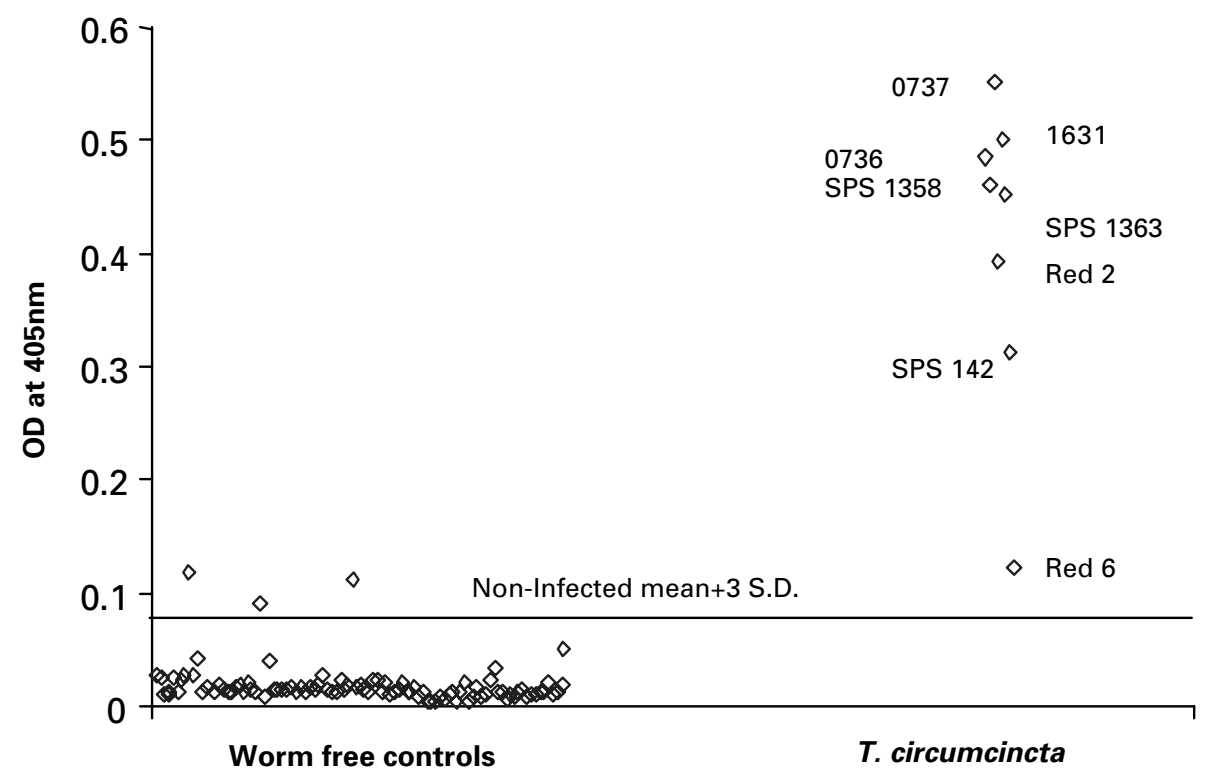

Fig. 2. Determination of the upper limit for the signal from non-infected animals. The values of OD readings from 98 faecal samples from worm-free lambs are compared with values obtained from 8 faecal samples from sheep experimentally infected with Teladorsagia circumcincta. The lettering and numbers next to the points representing infected animals are references to specific animals.

(signal: noise ratio $2 \cdot 3$ ) but not with other species. This assay was repeated 3 times, and on each occasion, similar results were obtained.

\section{Heat treatment of faecal supernatant to eliminate cross-reactivity}

Heat treatment of faecal supernatants from wormfree and $T$. circumcincta infected sheep revealed the presence of heat-sensitive material, with a partial loss of signal to $T$. circumcincta ( $28 \%$ reduction) following treatment at $100{ }^{\circ} \mathrm{C}$ for $5 \mathrm{~min}$. However, there was less impact on the signal: noise ratio which only fell from $16 \cdot 6$ to $14 \cdot 4$ (data not illustrated). Further direct comparative tests on faecal supernatants gave a reduction in the $\mathrm{OD}$ of samples from animals with
$H$. contortus from $0 \cdot 33$ to $0 \cdot 09$, compared with $0 \cdot 05$ to 0.04 for the worm-free animals but only 0.64 to 0.6 for an animal infected with $T$. circumcincta. Hence, whilst there was a reduction in the signal from all three samples, that from the animal carrying $T$. circumcincta remained considerably higher. We therefore explored further the efficacy of heat treatment in reducing cross-reactivity with other GI nematodes.

\section{Effect of duration of heat treatment of faecal supernatant}

In order to optimize the length of exposure to heat treatment at $100{ }^{\circ} \mathrm{C}$ (while preserving specific and eliminating cross-reactive signals), samples from sheep infected with $T$. circumcincta, $H$. contortus and 


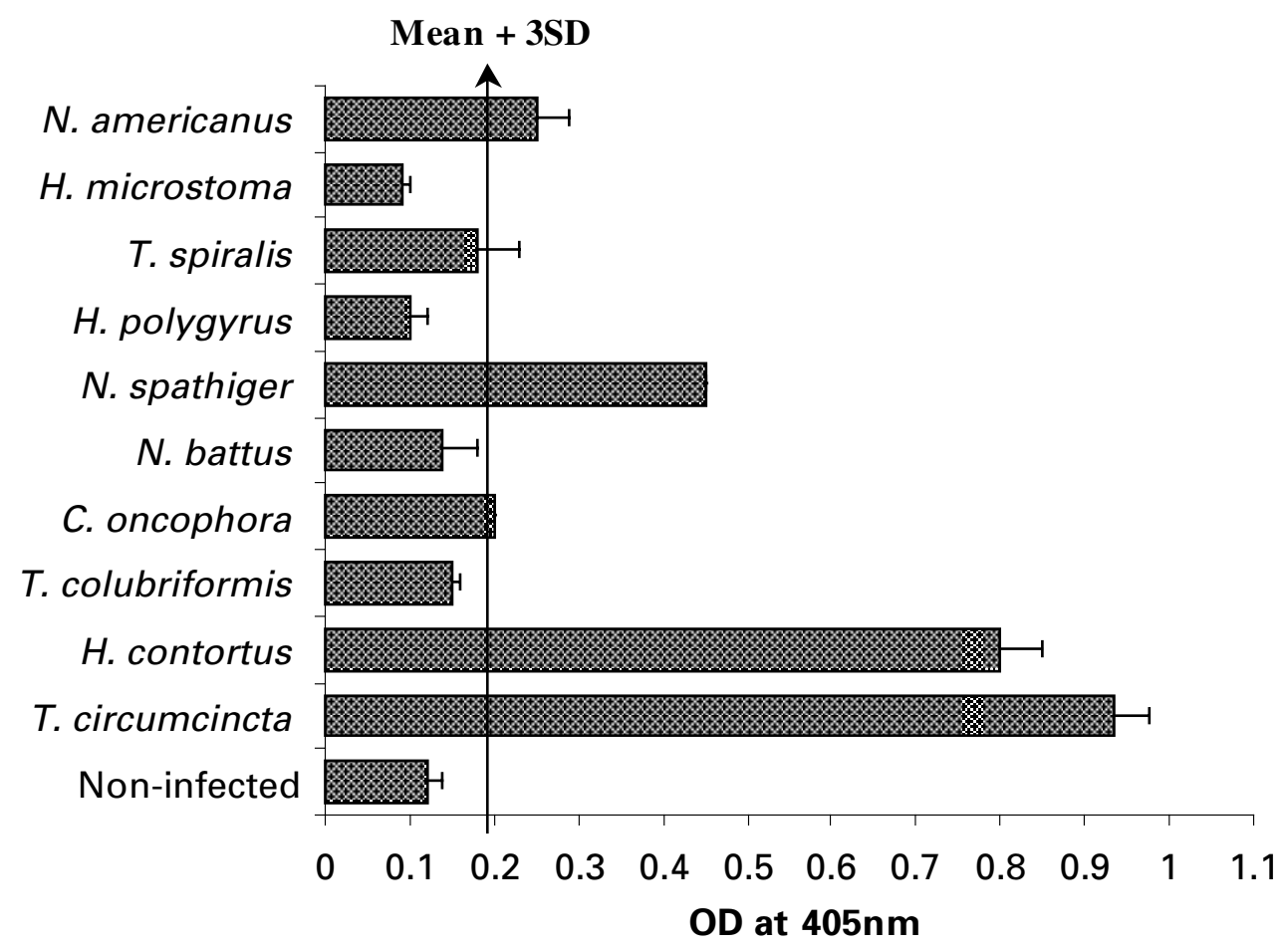

Fig. 3. Specificity of the copro-antigen capture ELISA, as determined through comparison of OD readings obtained from faeces from sheep and rodents experimentally infected with other species of GI nematodes and cestodes. The negative cut-off value $(0 \cdot 19)$ was calculated by the mean OD of the samples from worm-free sheep ( +3 s.D. $)$ and is indicated by the arrow across the figure. The mean values from the worm-free rodents are not illustrated on the graph, but were: mouse non-infected $\mathrm{OD}=0 \cdot 27$, hamster non-infected $\mathrm{OD}=0 \cdot 12$.

from worm-free animals were exposed to varying durations of heat treatment. A loss of signal occurred in all cases (data not illustrated); however, proportionally, the greatest loss of signal with time was in the samples from animals with $H$. contortus. After just $20 \mathrm{~min}$ of heat treatment, this was reduced to below background level. In contrast, the specific $T$. circumcincta signal to noise ratio was reduced from $17 \cdot 5$ to 5 after $20 \mathrm{~min}$, but was still very much higher than the threshold. Continued heat treatment after 20 min resulted in a further loss of specific activity to $T$. circumcincta, and the signal disappeared altogether after $60 \mathrm{~min}$. This protocol was repeated several times with some variation but essentially with similar results each time. Because a period of $20 \mathrm{~min}$ sufficed to enhance the difference between the signals from $T$. circumcincta and $H$. contortus, this was adopted as the optimal treatment and used thereafter in all assays.

\section{Effect of temperature on cross-reactivity of the assay}

Faecal supernatants were heat treated for $20 \mathrm{~min}$ at temperatures ranging from $21^{\circ} \mathrm{C}$ to $100{ }^{\circ} \mathrm{C}$ and then assayed. As expected, there was a loss of signal across all of the samples with heat treatment (data not illustrated) but in the case of $H$. contortus, the greatest loss was between $50{ }^{\circ} \mathrm{C}$ and $100{ }^{\circ} \mathrm{C}$, at which temperature the signal was reduced close to the negative threshold. At $100{ }^{\circ} \mathrm{C}$, the signal : noise ratios of faeces from $T$. circumcincta and $H$. contortus infected sheep were 11.7 and 1.9 , respectively. Overall, it was considered that a temperature of $100{ }^{\circ} \mathrm{C}$ was the simplest to implement, achieving an optimal balance between minimal loss of the specific signal, whilst essentially eliminating the major component of cross-reactivity with $H$. contortus and this was adopted in all future assays.

\section{Effect of a combined temperature of $100{ }^{\circ} \mathrm{C}$ for 20 min on specificity of the assay}

Before proceeding further, it was necessary to conduct a quantitative comparison. For this, all samples available from $T$. circumcincta $(n=12)$, and H. contortus $(n=6)$ infected sheep, together with 10 samples from naïve sheep, were tested in a direct comparison. The results are illustrated in Fig. 4A. There was a loss of signal in all samples tested, but the relative effect on the signal from $H$. contortus $(78 \cdot 8 \%$ reduction) was greater than that from $T$. circumcincta $(27 \cdot 0 \%$ reduction) and that from naïve animals (34.9\%). The cross-reactive signals from $H$. contortus were reduced in all but 2 cases to below the cut-off level and the 2 exceptions gave signals that were very close to the background level $(0 \cdot 118)$, at $0 \cdot 143$ and $0 \cdot 156$. Overall, heat treatment improved sensitivity from 66.7 to $85 \cdot 7 \%$, and specificity from $62 \cdot 5$ to $87 \cdot 5 \%$. 

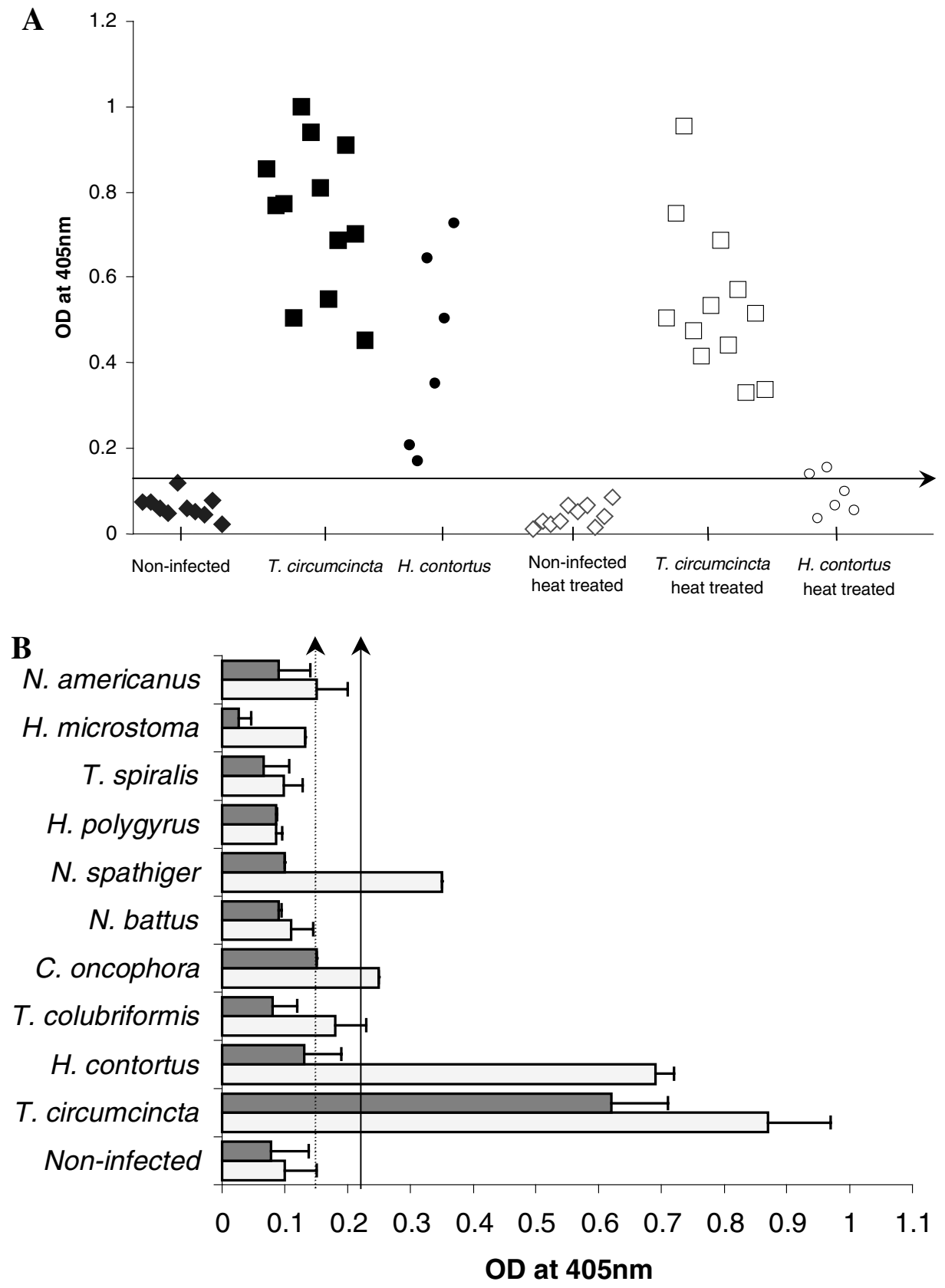

Fig. 4. (A) Quantitative assessment of the effect of subjecting faecal samples to heat treatment at $100{ }^{\circ} \mathrm{C}$ for 20 min. Values are OD values from individual sheep before and after heat treatment. A repeated measures ANOVA with heat treatment as the within-subject measure and infection as the between-subject measure gave a significant overall effect of heat treatment $\left(F_{1,25}=101 \cdot 6, P<0 \cdot 001\right)$, but also a significant interaction between heat-treatment and infection $\left(F_{2,25}=22 \cdot 7, P<0 \cdot 001\right)$ reflecting the proportionally much greater reduction in the signal from the Haemonchus contortus-infected sheep. There was also a significant main effect of infection $\left(F_{2,25}=57 \cdot 8, P<0 \cdot 001\right)$. (B) Specificity of the coproantigen capture ELISA before (pale columns) and after (filled in columns) heat treatment, as determined through comparison of OD readings obtained from faeces from sheep and rodents experimentally infected with other species of GI nematodes and cestodes. The negative cut-off value for sheep samples before heat treatment is shown by the solid line and that after heat treatment by the dotted line and both were calculated by the mean OD of the samples from worm-free sheep $(+3$ s.D.). The mean values from the worm-free rodents are not illustrated on the graph, but were: non-infected mouse, $O D=0 \cdot 28$ (heat treated $=0 \cdot 15$ ), non-infected hamster, $\mathrm{OD}=0 \cdot 19$ (heat treated $=0 \cdot 16$ ).

In a further assay, samples from animals infected with a range of species were re-examined. In each case, samples from infected animals were divided into 2 batches, one of which received no treatment, the other which was heat-treated for $20 \mathrm{~min}$ at $100^{\circ} \mathrm{C}$.
As Fig. 4B shows, the cross-reactive signals previously observed in $H$. contortus, and less so in $N$. spathiger, were again evident but after heat treatment were reduced to below the threshold level. By comparison, the specific $T$. circumcincta signal:noise 

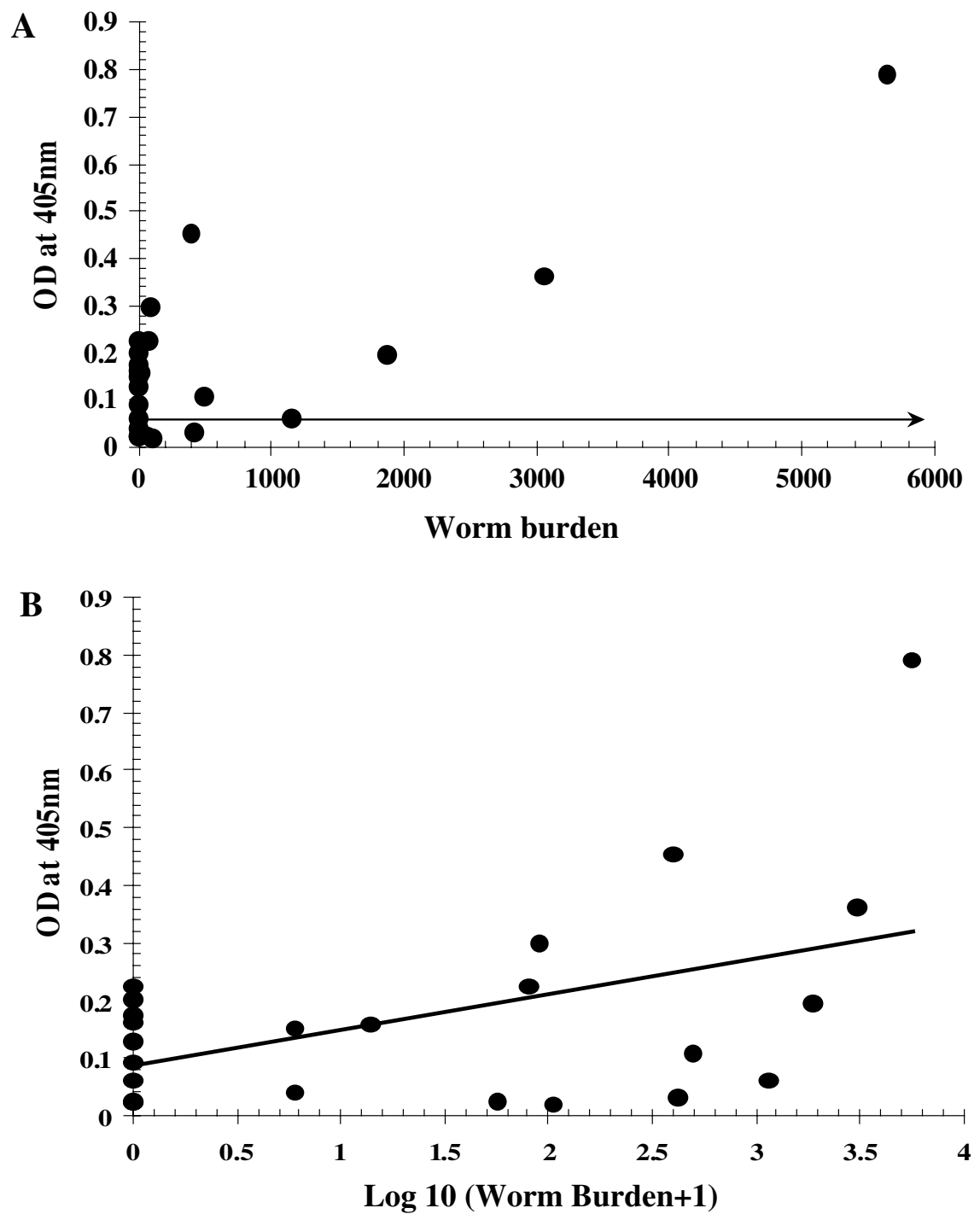

Fig. 5. The relationship between OD values and Teladorsagia circumcincta worm burdens in 23 sheep, obtained from a local abattoir in Nottingham. In (A), the raw data are given and the maximum threshold for the OD signal for uninfected status was 0.078 (horizontal line). (B) The worm burden data are expressed as Log 10 (total worms +1 ) and a line of best fit is shown to guide the eye.

ratio, was only reduced by heat treatment from $8 \cdot 8$ to $7 \cdot 9$.

\section{Relationship between $O D$ signal generated in} the coproantigen ELISA and the worm burden

Worm burdens from 23 naturally infected sheep obtained from a local abattoir ranged from zero to 5494 (no worms were recovered from 11 of the sheep but these may have harboured low-intensity worm burdens or arrested larvae). The relationship of the OD signal to worm burden is illustrated in Fig. 5. This was a significant positive relationship with $r_{s}=0 \cdot 472, n=23$ and $P=0 \cdot 023$. Although there was a clear dose-dependent relationship between worm burden and the signal from the $T$. circumcincta ELISA, at very low levels of infection, there was some discrepancy in the discrimination of positive and negative samples. However, there was no significant relationship between the OD signal and faecal egg counts. The coproantigen assay was repeated on the faecal samples on 3 separate occasions, and similar results were achieved on each occasion.

\section{DISCUSSION}

The results reported in this paper establish conclusively that a CC-ELISA for the detection of $T$. circumcincta is feasible and scientifically valid. Moreover, since the method was based on the conventional antigen-capture ELISA methodology, the approach lends itself to almost full automation after provision of faecal samples for analysis. Since in most countries domestic livestock are now routinely eartagged, rectal samples taken at initial roundup of the 
animals could be sent off for assessment and only those carrying confirmed heavy infestations, threatening productivity and welfare, would be treated with anthelmintic. Therefore, in pastoral countries where sheep are farmed in large flocks, routine preliminary diagnosis facilitating selective chemotherapy may become commercially viable.

In assessing possible cross-reactivity, we were constrained by available material and were not able to include other common GI nematodes of sheep in the UK, such as Chabertia ovina, Oesophagostomum venulosum and Trichostrongylus vitrinus, and therefore comprehensive evaluation of the assay against all likely species of worms still awaits completion. Nevertheless, among the species that were included, only 2 initially consistently generated cross-reactivity that was of concern. In this context, perhaps the most interesting contribution of this study to the development of CC-ELISAs for diagnosis of GI infections was the demonstration that a simple procedure, the temporary exposure of faecal supernatant to a high temperature, was beneficial in terms of reducing cross-reactivity with other ovine GI nematodes. The period of exposure to an optimum temperature of $100{ }^{\circ} \mathrm{C}$ was about $20 \mathrm{~min}$, and this treatment largely preserved the signal : noise ratio for the specific signal from $T$. circumcincta whilst essentially eliminating the cross-reactive signals from $H$. contortus and $N$. spathiger. Whilst the exact explanation for this phenomenon is not known, Johnson (1999) investigated its basis and concluded that heat treatment probably did not target glycosylated residues on E-S proteins and that protein moieties were more likely to be responsible. Clearly the epitopes in $\mathrm{E}-\mathrm{S}$ from $T$. circumcincta involved in specific recognition were more stable than those from other GI nematodes that generated the cross-reactive signals.

The assay was sensitive enough to detect $0 \cdot 25 \mu \mathrm{g}$ of parasite E-S per $\mathrm{ml}$ of faecal supernatant, much in line with earlier work on $H$. polygyrus and $S$. ratti in rodents and $O$. ostertagi in cattle, in each of which nanogram quantities of antigen were detected in CCELISAs (Nageswaren et al. 1994; Johnson et al. 1995; Agneessens et al. 2001). In terms of quantitative detection of worm burdens in sheep, we were limited by our reliance on abomasa and rectal specimens from the local abattoir. These animals will have been exposed to a range of GI nematodes typical for the region and will have carried worms of each in different stages of development. Since only the abomasa were examined for worms, no information was available about the degree of infection with other species and hence clearly this aspect still awaits more comprehensive evaluation, perhaps under full field trial conditions. Nevertheless, animals with 1000 or more $T$. circumcincta produced strong OD signals that could be considered reliably as indicative of significant infection and the need for treatment. Those with fewer worms produced more variable signals, but it may also be pertinent that immunity to $T$. circumcincta infections in sheep acts primarily to reduce the fecundity and length of adult worms, rather than to induce loss of adult worms (Stear et al. 1995; Strain et al. 2002), and it may be that worms affected by the initial immune response also secrete less $\mathrm{E}-\mathrm{S}$.

Since in some parts of the world sheep husbandry has become very difficult as a consequence of the failure of all available classes of anthelmintics to control worm burden (van Wyk, 1990), the selective treatment that would become feasible with the availability of this assay might be one way of slowing down this trend. Moreover, by concentrating treatment on heavily infected animals, some of the costs of diagnosis would be offset by savings on the cost of anthelmintics. In terms of benefits on the broader front, the larger part of the in vivo reservoir of infection would be eliminated and the untreated animals, carrying lower worm burdens, would have an opportunity to develop natural immunity to re-infection. Thus, selective dosing of animals harbouring heavy infections, and judicious use of anthelminthic drugs, may delay the onset of anthelminthic resistance in regions where it has not yet appeared and may slow the spread in those countries where it is already a significant problem.

The work described here was conducted with the chief objective of developing a specific assay for $T$. circumcincta, but in practical terms an assay merely capable of distinguishing sheep that carry heavy total worm burdens (irrespective of species) may be more useful to the livestock owners. This could be achieved either by the use in parallel of a number of specific assays or by developing an assay based on appropriate proportions of specific sera to detect the mix of species in a given geographical region. Another immunological approach would be to develop monoclonal antibodies for the E-S of each of the important species, as has already been done for H. contortus (Ellis et al. 1993), although the reported sensitivity in this case was low. These would be used in a mix or as specific assays in parallel. For human GI nematodes, dip sticks may be relevant, much as in the detection of human cestode infections (Allan et al. 1993). However, for large-scale farming of domestic animals, an automated CC-ELISA in the hands of an agricultural diagnostic service provider would be the most likely way forward. Now that CC-ELISA assays have been demonstrated as feasible for $O$. ostertagi and for $T$. circumcincta, comparable assays for H. contortus and Trichostrongylus spp. require development, as well as perhaps a general (higher taxa) assay for all trichostrongyloid GI nematodes. In practice, a logical compromise may be to implement a 2-step diagnostic service, with detection of animals carrying heavy GI nematode (trichostrongyloid) infections as the first step, followed by species-specific CC-ELISA if warranted. 
D. A. J. was supported by a post-graduate studentship from the Ministry of Agriculture, Food and Fisheries, for which we are extremely grateful. We are also indebted to Drs R. Coop, F. Jackson, D. Knox and D. Bartley of the Moredun Research Institute, staff at Compton Animal Health and D. Nicholas at Pfizer Animal Health, for the provision of faeces from either infected or worm-free animals.

\section{REFERENCES}

AGNeEssens, J., CLAEREbout, E. \& Vercruysse, J. (2001). Development of a copro-antigen capture ELISA for detecting Ostertagia ostertagi infections in cattle. Veterinary Parasitology 97, 227-238.

ALlan, J. C., CRAig, P. S., GARCIA NOVAL, J., MENCOS, F., LIU, D., WANG, Y., WEN, H., ZHOU, P., STRINGER, R., ROGAN, M. \& ZEYHLE, E. (1992). Coproantigen detection for immunodiagnosis of echinococcosis and taeniasis in dogs and humans. Parasitology 104, 347-355.

ALLAN, J. C., MENCOS, F., GARCia NOVAL, J., SARTI, E., FLISSER, A., WANG, Y., LIU, D. \& CRAIG, P. S. (1993). Dipstick dot ELISA for the detection of Taenia coproantigens in humans. Parasitology 107, 79-85.

ALlan, J. C., VELASQUEZ-TOHOM, M., TORRES-ALVAREZ, R., YURRITA, P. \& GARCIA-NOVAL, J. (1996). Field trial of the coproantigen based diagnosis of Taenia solium taeniasis by enzyme linked immunosorbent assay. American Fournal of Tropical Health and Hygiene 54, 352-356. BAUMANN, D. \& GOTTSTEIN, B. (1987). A double antibody sandwich ELISA for the detection of Entamoeba histolytica in stool samples of humans. Tropical Medicine and Parasitology 38, 81-85.

BRADFORD, M. M. (1976). A rapid and sensitive method for the quantification of microgram quantities of protein utilising the principle of protein-dye binding. Analytical Biochemistry 72, 248-254.

COLES, G. C. (1995). Chemotherapy of human nematodes: learning from the problems of sheep. Fournal of the Royal Society of Medicine 88, 649P-651P.

CONDOR, G. A. \& CAMPBELL, W. C. (1995). Chemotherapy of nematode infections of veterinary importance, with special reference to drug resistance. Advances in Parasitology 35, 1-84.

DEPLAZES, P., GOTTSTEIN, B., ECKERT, J., JENKINS, D. J., EWALD, D. \& JIMINEZ-PALACios, s. (1992). Detection of Echinococcus coproantigens by enzyme-linked immunosorbent assay in dogs, dingoes and foxes. Parasitology Research 78, 303-308.

ELlis, T. M., GREGORY, A., TURNOR, R., KALKHOVEN, M. \& Wroth, R. H. (1993). Detection of Haemonchus contortus surface antigen in faeces from infected sheep. Veterinary Parasitology 51, 85-97.

Galen, R. S. \& GAMBINo, S. R. (1975). Beyond Normality: The Predictive Value of Medical Diagnosis. Wiley, New York.

GREEN, E. L. (1986). Immunological detection of parasite antigen in faeces. Parasitology Today 2, 198-200.

GREEN, E. L., WARHURST, D. C. \& MILES, M. A. (1985). Immunodiagnostic detection of Giardia antigen in faeces by a rapid visual enzyme linked immunosorbent assay. The Lancet 2, 691-694.

HENNESSY, D. R. (1997). Livestock parasite treatment - a call for greater interaction between research and industry sectors. International Fournal for Parasitology 27, 1129-1133.
JACKSON, F. \& COOP, R. L. (2000). The development of anthelmintic resistance in sheep nematodes. Parasitology 120, S95-S107.

JACKSON, F., JACKSON, E. \& COOP, R. L. (1992). Evidence of multiple anthelminthic resistance in a strain of Teladorsagia circumcincta (Ostertagia circumcincta) isolated from goats in Scotland. Research in Veterinary Science 53, 371-374.

JELENIK, T., PEYERL, G., LOSCHER, T. H. \& NOTHDURFT, H. D. (1996). Giardiasis in travellers: evaluation of an antigencapture ELISA for the detection of Giardia lambliaantigen in stool. Zeitschrift für Gastroenterologie 34, 237-240.

JOHnson, D. A. (1999). Coproantigen capture ELISA for the detection of intestinal nematode infections. Ph.D. thesis. School of Biological Sciences, University of Nottingham.

JOHNSON, D. A., BEHNKE, J. M. \& COLES, G. C. (1999). Development of a coproantigen capture ELISA for the detection of Teladorsagia circumcincta in sheep. In Proceedings of the 17th WAAVP Conference, Copenhagen, Denmark.

JoHnson, M. (1994). Coproantigen ELISA for GI nematodes. Ph.D. thesis. School of Biological Sciences, University of Nottingham.

JOHNSON, M. J., BEHNKE, J. M. \& COLES, G. C. (1995). Detection of gastrointestinal nematodes by a coproantigen capture ELISA. Research in Veterinary Science 60, 7-12.

NAGESWAREN, C., CRAIG, P. S. \& DEVANEY, E. (1994). Coproantigen detection in rats experimentally infected with Strongyloides ratti. Parasitology 108, 335-342.

NICHOLLS, J. \& OBENDORF, D. L. (1994). Application of a composite faecal egg count procedure in diagnostic parasitology. Veterinary Parasitology 52, 337-342.

RODRIGUEZ-CANUL, R., FRASER, A., ALLAN, J. C., DOMINGUEZ-ALPIZAR, J. L., ARGAEZ-RODRIGUEZ, F. \& CRAIG, P. S. (1999). Epidemiological study of Taenia solium taeniasis/cysticercosis in a rural village in Yucatan state, Mexico. Annals of Tropical Medicine and Parasitology 93, 57-67.

roepstorff, A. (1998). Natural Ascaris suum infections in swine diagnosed by coprological and serological (ELISA) methods. Parasitology Research 84, 537-543. SMITH, G. (1990). Chemotherapy: future problems. In Hookworm Disease. Current Status and New Directions (ed. Schad, G. A. \& Warren, K. S.), pp. 291-303. Taylor \& Francis, London.

STEAR, M. J., BISHOP, S. C., DOLIGALSKa, M., DUNCAN, J. L., HOLMES, P. H., IRVINE, J., McCRIRIE, L., McKellar, Q. A., SINSKI, E. \& MURRAY, M. (1995). Regulation of egg production, worm burden, worm length and worm fecundity by host responses in sheep infected with Ostertagia circumcincta. Parasite Immunology 17, 643-652.

STEAR, M. J. \& MURRAY, M. (1994). Genetic resistance to parasitic disease: particularly of resistance in ruminants to gastrointestinal nematodes. Veterinary Parasitology 54, 161-176.

STRAin, S. A. J., BISHOP, S. C., HENDERSON, N. G., KERR, A., McKellar, Q. A., Mitchell, S. \& STEAR, M. J. (2002). The genetic control of IgA activity against Teladorsagia circumcincta and its association with parasite resistance in naturally infected sheep. Parasitology 124, 545-552. 
URQUHART, G. M., ARMOUR, J., DUNCAN, J. L., DUNN, A. M. \& Jennings, F. w. (1987). Veterinary Parasitology. Avon: Longman Scientific \& Technical.

VAN WYK, J. A. (1990). Occurrence and dissemination of anthelmintic resistance in South Africa, and management of resistant worm strains. In Resistance of Parasites to Antiparasitic Drugs (ed. Boray, J. C., Martin, P. J. \& Roush, R. T.), pp. 103-113. MSD, AGVET, Rahway, New Jersey, USA.
WALler, P. J. (1986). Anthelmintic resistance in nematode parasites of sheep. Agricultural Zoology Reviews 1, 333-373.

WALLER, P. J. (1993). Control strategies to prevent resistance. Veterinary Parasitology 46, 133-142.

WALLER, P. J., DASH, K. M., BARGER, I. A., LE JAMBRE, L. F. \& PLANT, J. (1995). Anthelminthic resistance in nematode parasites of sheep: learning from the Australian experience. Veterinary Record 136, 411-414. 